II.

\title{
A Case of Endothelioma of the Uterus arising in a Fibro-myoma, and associated with Glandular Car- cinoma of the Endometrium.*
}

\author{
By Mrues H. Priluirs, M.B., B.S. (Lond.), F.R.C.S. (Eng.), \\ Assistant Surgeon to the Jessop Hospital for Women, Sheffeld.
}

The specimen, described in this paper, was originally shown as an instance of the invasion of a submucous fibro-myoma of the uterus by a mixed-celled sarcoma. Professor Beattie, of the University of Sheffield, has since made a very thorough microscopical investigation of the specimen, and has come to the following conclusions :

1. The tumour is an endothelioma arising in the lymphatics of a fibro-myoma.

2. The glands of the endometrium, adjoining the growing edge of the endothelioma, have undergone malignant proliferation, forming an independent columnar-celled carcinoma.

3. The appearances of the growths are, in many places, modified by the presence of inflammatory cells.

I am greatly indebted to him for his assistance and for the pathological report which is subjoined.

\section{Climical Report.}

The patient, 50 years of age, was admitted into Mr. Favell's wards, whilst I was doing his holiday duty, and I have to thank him for permission to report the case.

Though married for ten years she had never been pregnant. She had been a widow for fifteen years.

For nine years she had suffered from increasing menorrhagia with severe hypogastric cramp-like pain during the periods; for fourteen months there had been intermenstrual hæmorrhage with frequent floodings during which the cramp-like pains were always worse.

For several months there had been a constant, very offensive, vaginal discharge. Constant backache and steady loss of flesh and strength were the only other symptoms.

She was thin and anæmic, but not cachectic; her temperature, normal or subnormal in the mornings, reached $100^{\circ}$ and $101^{\circ}$ at

- Based on a communication brought before a meeting of the North of England Obstetrical and Gynecological Society, beld November 1907, when the specimon was shown. 
night. There was a profuse offensive vaginal discharge generally purulent in appearance at times blood-stained and containing small pieces of necrotic material.

The external os was found to be dilated, but not sufficiently to admit the index finger, and the uterus was uniformly enlarged to the size of a four-monthe' pregnancy. No other tumour could be detected on bimanual examination, but there was considerable tenderness on each side of the uterus.

Suspecting a submucous fibroid, infected and becoming gangrenous, or possibly associated with malignant disease of the endometrium, I explored the uterus three days after admission.

The cervix was easily dilated and, with the gloved finger a large quantity of stinking necrotic material was easily removed; this, from its soft, non-fibrous, brain-like characters, suggested a malignant growth rather than a fibro-myoma. Just above the internal os a firm, rounded tumour, the size of a golf-ball, projected into the uterine cavity. It was now found that the mobility of the uterus was deficient, especially as regardy descent on traction, and, as there was no parametric infiltration, the presence of peritoneal adhesions was suspected.

After free irrigation with biniodide solution, the uterine cavity was packed with 5 per cent. formalin gauze. The temperature rose to $103 \cdot 4^{\circ}$ that night, but fell to and remained at normal for thirty-six hours afterwards, and the vaginal discharge was less in amount and not offensive.

The material removed was examined microscopically and reported to be sarcomatous, and so, three days after the exploration, I closed the external os by sutures, cleansed the vagina, opened the abdomen and removed the uterus, entire, with its appendages; both Fallopian tubes were thickened and their ostia closed, and the left was firmly adherent to the pelvic peritoneum and the pelvic colon. I left the vagina open, united the pelvic peritoneum by a purse-string suture, and closed the abdominal wound in three layers without drainage.

The temperature rose to $101^{\circ}$ that night, fell to normal next morning, and there remained until the fourth night, when it rose to $101^{\circ}$, never to reach the normal again; for four weeks it fluctuated between $100^{\circ}$ and $104^{\circ}$, although some suppuration in the lower part of the abdominal wound was easily controlled, and no inflanmatory exudation could be felt in the pelvis, and there were no signs of peritonitis.

In spite of the absence of rigors, septic thrombosis of pelvic veins was suspected, and this suspicion was supported by the occurrence of swelling of the right leg and thigh four weeks after the operation; a few days later the patient suddenly died.

At the autopsy, septic thrombosis of the right external and of both internal iliac veins, and an embolus in the left pulmonary artery 


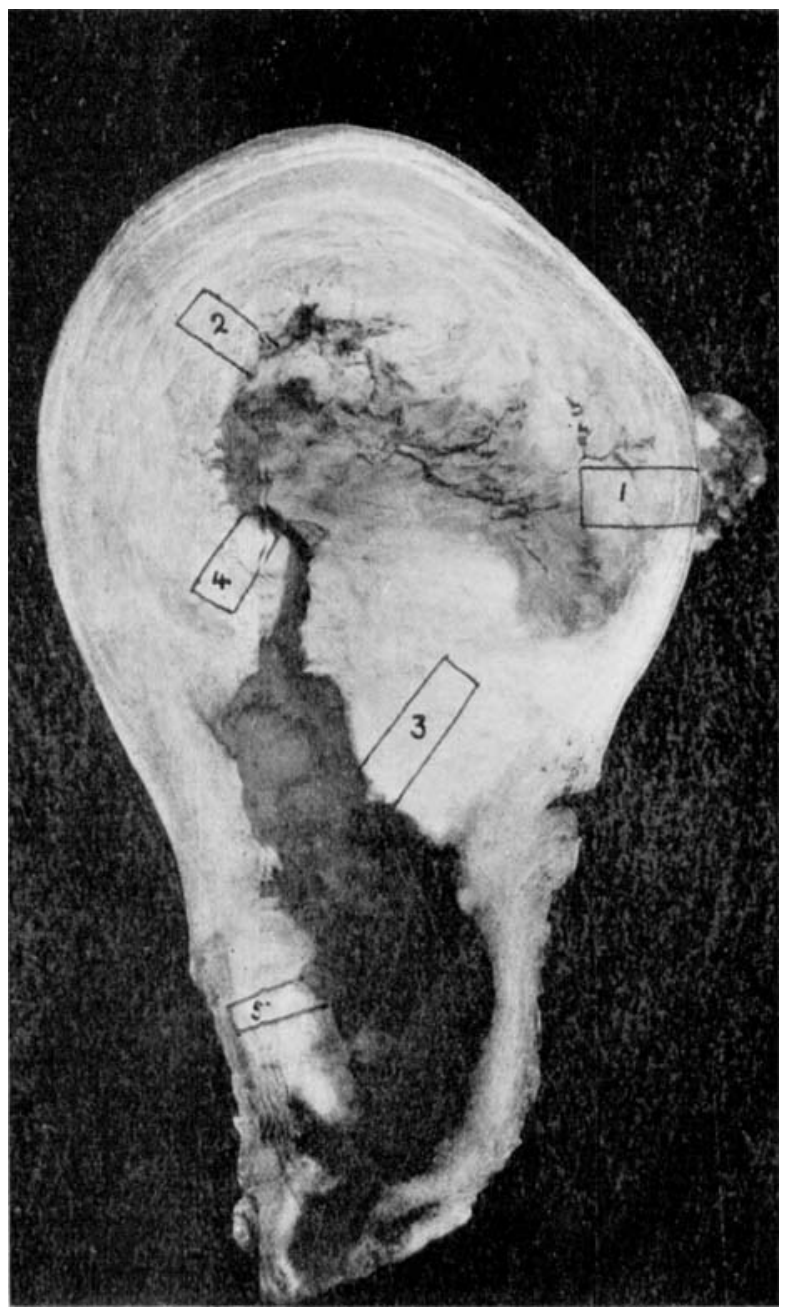

Photograph of the inner surface of the left half of the uterus. The areas marked out and numbered represent the pieces of material removed. from the other half of the tumour, for microscopical examination. The onter end of the left Fallopian tube projects beyond the anterior surface of the uterus. 
were found. There was a small collection of grumous pus in the subperitoneal connective tissue around the right pelvic veins, but no peritonitis. There were no secondary growths.

\section{Pathological Report.}

Macroscopical. The specimen consists of the left half of the vertically bisected uterus, which, after hardening, and before division, measured $12.75 \mathrm{~cm}$. in length, $7.5 \mathrm{~cm}$. in width, and $7 \mathrm{~cm}$. in its greatest antero-posterior diameter. The anterior wall is greatly thickened by a mass of new growth, which bulges into and almost obliterates the upper part of the uterine cavity. It has also deeply eroded the true fibro-muscular wall to within, at one place, $2.5 \mathrm{~mm}$. of the peritoneal coat. This growth presents two strikingly distinct portions: (a) an upper one : in colour greyish, with reddish-brown areas of hæmorrhage, friable, deeply fissured, eroding the uterine wall in front, presenting a ragged necrotic surface to the uterine cavity, and capping (b) the lower growth, which is ovoid in shape $(3.5 \times 2.5 \mathrm{~cm}$.), yellowish-white, of firm consistence and smoothly rounded on its free surface. The posterior wall is thickened to a less degree, though there is, near its middle point, a considerable projection into the uterine cavity. (It is here that malignant proliferation of the endometrial glands is to be found.) The cervix is slightly elongated, its cavity dilated (by the gauze packing), and its wall thinned, except for an ovoid swelling $(2 \times 0.8 \mathrm{~cm}$.) in the upper twothirds of the posterior wall. The Fallopian tube is tortuous and thickened, its abdominal ostium closed, and its outer fourth distended by pus. The ovary, adherent to the tube, is normal in appearance. (The right appendages presented similar changes.)

Microscopical (Professor Beattie). The tumour is largely composed of irregular masses of cells lying between the muscular and fibrous bundles of the uterus, and of the fibroid in which it seems to have originated. The cells are mainly polyhedral in shape, but there are also numerous rounded and spindle-shaped ones (Section 1). At different areas in the tumour the classes of cells vary considerably. At some places, spindle-shaped cells largely predominate, at other parts polyhedral cells alone are found. In both classes there is a well-defined nucleus, moderately rich in chromatin, but showing quite clearly the nuclear membrane, nuclear network and nucleolus. In their pathological structure the cells more closely resemble the carcinomatous type than the sarcomatous. In their general arrangement they vary at different areas. At some parte they are closely packed together with a very small amount of intercellular supporting tissue, and give no indication of their site of origin; at other parts they are more or less closely packed in irregular spaces, which sometimes show a flattened endothelial lining. In these irregular spaces, 
which we consider to be lymphatics, there is no intercullular material, and at places there is a distinct proliferation of the lining endothelium. At certain parts, and especially near the growing margins of the tumour, the cells are arranged in a definitely concentric fashion round both large and small blood-vessels, and this appearance combined with the morphological characters of the cells, and the great amount of growth in lymphatic spaces, leads to the view that the tumour is of the nature of an endothelioma, and that it has arisen by an abnormal proliferation of the endothelial cells lining the lymphatic spaces. (Section 2.) It has spread by direct invasion of the tissues with separation and destruction of both muscle and fibrous bundles. The tumour seems to have arisen in and largely destroyed a uterine fibroid, and from this focus it has spread along the lymphatics to distant parts of the uterus. Many of the lymphatic channels are filled with the tumour cells, and some of them show well-marked proliferation of their lining endothelium. There is very marked invasion of the tumour by inflammatory cells, which are probably secondary to the septic condition of the endometrium, and in some cases there is well-defined necrosis of the tumour cells, the nucleus having lost its capacity for taking up basic dyes, and the cells having become fused into irregular homogeneous masses, which stain with eosin more brightly than the other parts of the tumour. At one part, apparently just at the growing edge of the tumour, on the posterior wall of the uterus, there is a very definite proliferation of glandular structure. This presents the typical appearance of an adeno-carcinoma. The gland spaces are very irregular in shape and size, and show numerous papilliform ingrowths. No definite basement membrane can be detected. They are separating and causing a certain degree of atrophy of the muscle fibres. It is possible that they are abnormal projections of a glandular endometritis, their great and irregular proliferation being due to the irritation of the tumour growth. Their very deep position in the wall is rather against this view, and I am inclined to regard this growth as an independent tumour of the type of a localised columnar-celled carcinona. The nodule in the posterior cervical wall, is composed of cells similar in character to those of the main tumour; it is evidently a metastatic deposit.

The main tumour is of special interest from the point of view of histological diagnosis. In certain areas the microscopical appearances are practically indistinguishable from those of a mixed-celled sarcoma; while in other parts the structure more closely resembles that of an epithelial or endothelial tumour. As already stated, I am of opinion that the tumour should be placed among the endotheliomata. On this view the variation in histological appearances is not surprising, when we consider the origin of the endothelium of the lymphatics, and the fibrous and muscular tissues. The primitive 
mesoblast becomes differentiated into mesothelium and mesenchyme, and it is from the latter that the endothelium of blood-vessels and lymphatics, as well as the fibrous connective tissues, and the unstriped muscular tissues arise.

Thus both sarcomata and endotheliomata take origin from this mesenchyme - the former probably, however, at an earlier and more primitive stage of development. It is not therefore surprising that, in a malignant new growth arising from endothelium, there should be at parts a reversion to the more primitive sarcomatous structure, and this would, $I$ think, be more liable to occur if the original malignant neoplasm were growing in a fibroid tumour, where presumably the various structures would, at any rate in their life history, be less fully and less perfectly developed than the original tissues of the uterus.

Further, this alteration in type of tumour, even of those of distinct embryogenetic origin, has been shown to occur experimentally in mice by Ehrlich, Bashford and other-malignant epithelial tumours having become definite sarcomata. 